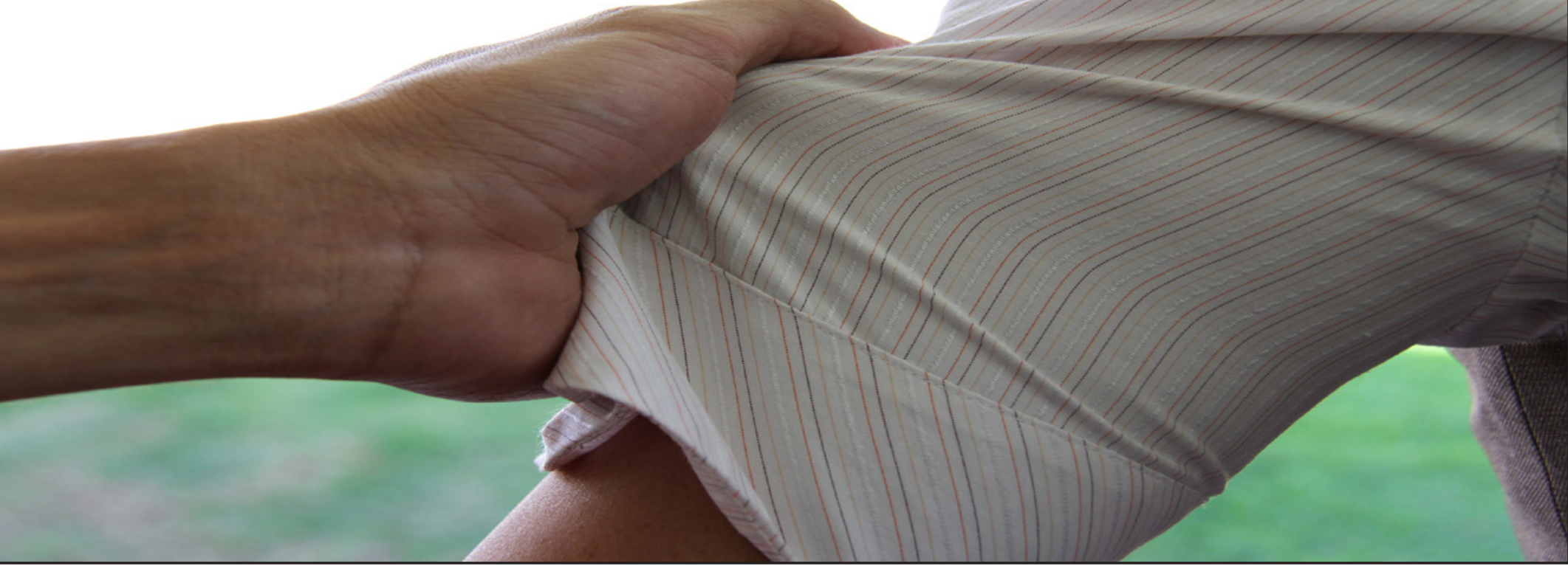

\title{
VIOLENCIA EN EL NOVIAZGO, ESCUELA PREPARATORIA PÚBLICA DE SONORA.
}

DATING VIOLENCE, PUBLIC HIGH SCHOOL OF SONORA.

\begin{tabular}{r} 
GLORIA CECILIA VERDUGO-LEÓN.* \\
\hline MARÍA AMAVIZCA-VELÁSQUEZ. ** \\
MARÍA FERNANDA SUAREZ. *** \\
JOANNA MARTÍNEZ. ${ }^{* * * *}$
\end{tabular}

OLIMPIA OFELIA CORTEZ-RIVERA. *****

(*) Estudiante de Enfermería. Departamento de Enfermería. Universidad de Sonora. Email: ceci_vele@hotmail.com

$\left.{ }^{(* *}\right)$ Estudiante de Enfermería. Departamento de Enfermería. Universidad de Sonora. Email: mariiacu05@hotmail.com

${ }^{(* * *)}$ Estudiante de Enfermería. Departamento de Enfermería. Universidad de Sonora.

${ }^{(* \star *)}$ Estudiante de Enfermería. Departamento de Enfermería. Universidad de Sonora. Email: feer_suarez394@hotmail.com

${ }^{* * * * *)}$ Maestra en Ciencias. Docente de Asignatura. Departamento de Enfermería. Universidad de Sonora. Email: Olimpia. cortez@enfermeria.uson.mx

\section{RESUMEN:}

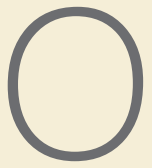

bjetivo: Identificar la prevalencia de violencia en las relaciones de noviazgo de los estudiantes de una preparatoria de la ciudad de Hermosillo. Es un estudio de tipo exploratorio, descriptivo y transversal que contó con la participación de 214 estudiantes del turno matutino de los tres diferentes semestres de la preparatoria. Para la recolección de datos se utilizó una encuesta realizada por Guzmán, validada por Instituto Aguascalientes de las mujeres. Se obtuvo como resultado que de la muestra encuestada el $44.3 \%$ de estos alumnos presentan un grado de violencia. La mayoría de los jóvenes (29.4\%) indican señales iniciales de violencia, un $10.7 \%$ se identificaron con una relación de abuso en el noviazgo, otro 3.3\% mencionó estar en una relación de abuso severo, por ultimo un $0.9 \%$ presentó una relación violenta. Del $100 \%$ de los hombres participantes (110) el $47 \%$ sufre algún tipo de violencia en el noviazgo, observando que este género es más violentado que el género femenino, ya que de un $100 \%$ de ellas (104) el $41 \%$ indicó presentar algún grado de violencia. Conclusión: Es necesario conocer el porcentaje de violencia en el noviazgo para poder afrontar este problema que afecta la salud mental de los estudiantes, por lo que en base a los resultados encontrados se intervino en base al diagnóstico de enfermería Riesgo de Violencia Dirigida a otros (00138).

Palabras claves: Violencia, citas, adolescente 


\section{ABSTRACT}

Objective: To identify the prevalence of violence in dating relationships of students from a high school in the city of Hermosillo. It is an exploratory, descriptive and cross type with the participation of 214 students in the morning shift of three different semesters of high school. A survey conducted by Guzman, Aguascalientes Institute validated by women was used for data collection. As a result of the survey shows that $44.3 \%$ of these students have a degree of violence. Most young people $(29.4 \%)$ indicate early signs of violence, $10.7 \%$ were identified in an abusive relationship dating another 3.3\% reported being in a relationship of severe abuse, finally $0.9 \%$ had a violent relationship . $100 \%$ male participants (110) $47 \%$ suffer some form of dating violence, noting that this genre is that the female gender violated because $100 \%$ of them (104) $41 \%$ reported moderate some degree of violence. Conclusion: It is necessary to know the percentage of dating violence to deal with this problem that affects the mental health of students, according to the results were executed an intervention based on nursing diagnosis Risk of violence directed at others (00138).

Keywords: Violence, Dating, Teen

\section{INTRODUCCIÓN}

La violencia contra la mujer constituye un grave problema de salud pública y una violación de los derechos humanos de las mujeres. La Organización Mundial de la Salud (OMS) define violencia como el uso deliberado de la fuerza física o el poder, ya sea en grado de amenaza o efectivo, contra uno mismo, otra persona o un grupo o comunidad, que cause o tenga muchas probabilidades de causar lesiones, muerte, daños psicológicos, trastornos del desarrollo o privaciones. Las cifras recientes de la prevalencia mundial indican que el $35 \%$ de las mujeres del mundo han sufrido violencia de pareja o violencia sexual por terceros en algún momento de su vida.(1)

Según la OMS, 3 de cada 10 adolescentes denuncian que sufren violencia en el noviazgo. Muchas de las mujeres que son maltratadas durante el matrimonio vivieron violencia en el noviazgo y no la identificaron. La violencia más frecuente es la ejercida por el actual o último esposo o compañero, declarada por el $43.2 \%$ de las mujeres, le sigue la violencia en la comunidad por el $39.7 \%$ de las mujeres; la violencia en el trabajo representa el $29.9 \%$ y la escolar 15.6\%".6. (2)

En México se realizó la Encuesta Nacional sobre la Dinámica sobre la Relaciones en Hogares (ENDIREH), donde el 47\% de las mujeres de 15 años y más sufrió de algún acto de violencia por parte de su pareja durante su última relación. Por entidad federativa, obtuvo que el nivel de violencia de pareja a lo largo de la relación con mayor prevalencia es el Estado de México con 57.6\%, le sigue Nayarit (54.5\%), Sonora (54.0\%), el Distrito Federal (52.3\%) y Colima (51.0\%). En Sonora, un total de 333560 mujeres de 15 años y más que habitan en este estado declararon haber sufrido violencia a lo largo de su última relación por parte de su pareja o su ex pareja. De las mujeres que residían en Sonora de 15 y más años, $14.9 \%$ han sufrido violencia física; de estas 51 de casa 100 tienen entre 35 y 54 años y 35 de cada 100 pertenecen al grupo más joven, se ubican en el grupo de 15 a 34 años. ${ }^{(3)}$

La violencia en el noviazgo merece especial atención, sobre todo, cuando se inician las relaciones entre los jóvenes y se definen roles y límites. Todos los seres humanos requieren fomentar relaciones interpersonales positivas. En estos casos la enfermera actúa como agente de cambio fomentando la interacción interpersonal entre los usuarios, siendo el noviazgo entre los adolescentes un primer acercamiento a lo que será formar un vínculo familiar, de ahí que sea tan importante que desde ese momento las relaciones interpersonales sean positivas.

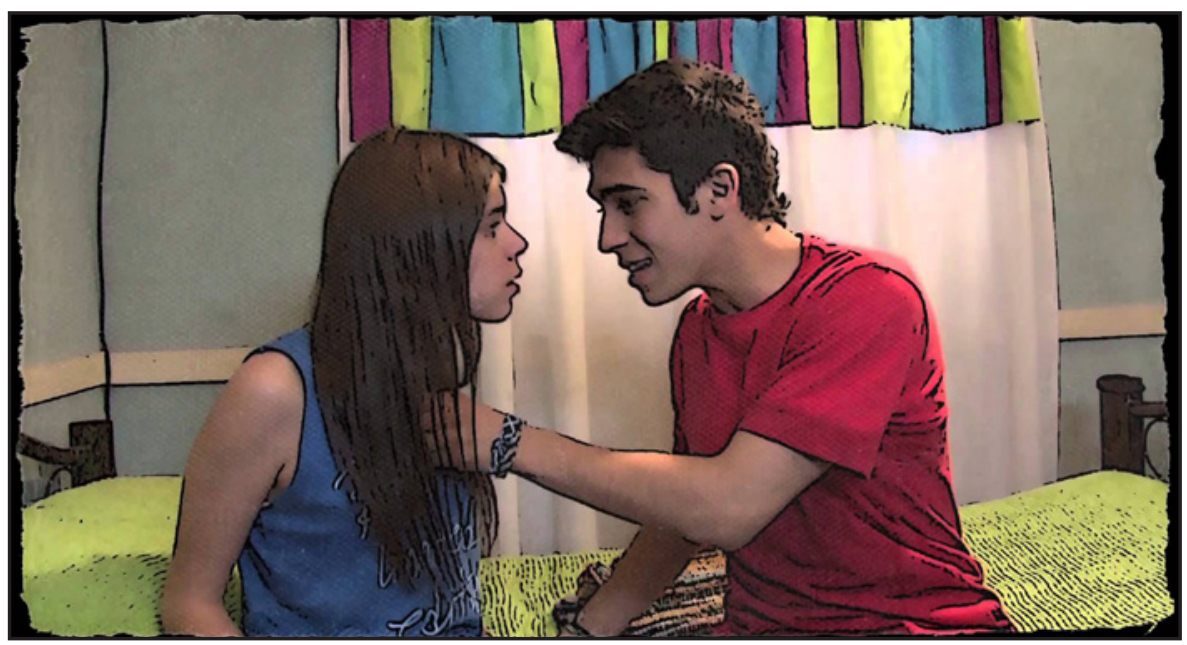




\section{MARCO REFERENCIAL}

\section{Violencia en el noviazgo}

El noviazgo es una vinculación que se establece entre dos personas que se sienten atraídas mutuamente. Representa una oportunidad para conocerse, una etapa de experimentación y de búsqueda, con actividades, gustos y pensamientos en común, y es un preámbulo para una relación duradera. ${ }^{(2)}$

Las relaciones de pareja son uno de los principales recursos de apoyo social que contribuyen al bienestar psicosocial y al afrontamiento de situaciones estresantes en la adolescencia y la juventud, por lo que el establecimiento de relaciones íntimas juega un papel muy importante en el desarrollo socioemocional de las personas. Además, el ajuste de una persona adulta es en función de la capacidad de iniciar y mantener una relación amorosa con otra. ${ }^{(4)}$

La violencia se define como el uso intencional de la fuerza o el poder físico, de hecho o como amenaza, contra uno mismo, otra persona, un grupo o comunidad, que cause o tenga muchas probabilidades de causar lesiones, muerte, daños psicológicos, trastornos del desarrollo o privaciones. El noviazgo es el periodo de vida en el que dos personas establecen una relación afectiva con la intención de conocerse mejor y, en su caso institucionalizarla de manera permanente, durante el cual se establecen relaciones amorosas libremente consentidas y mutuamente aceptadas.

La violencia en el noviazgo es todo ataque intencional de tipo sexual, físico o psíquico, de un miembro de la pareja contra el otro en una relación de noviazgo con el objeto de controlar o dominar a la persona. Las conductas violentas en las relaciones de pareja no son percibidas como tales, por las víctimas, o por los agresores, se confunden con muestras de afecto, que en realidad ocultan conduc-

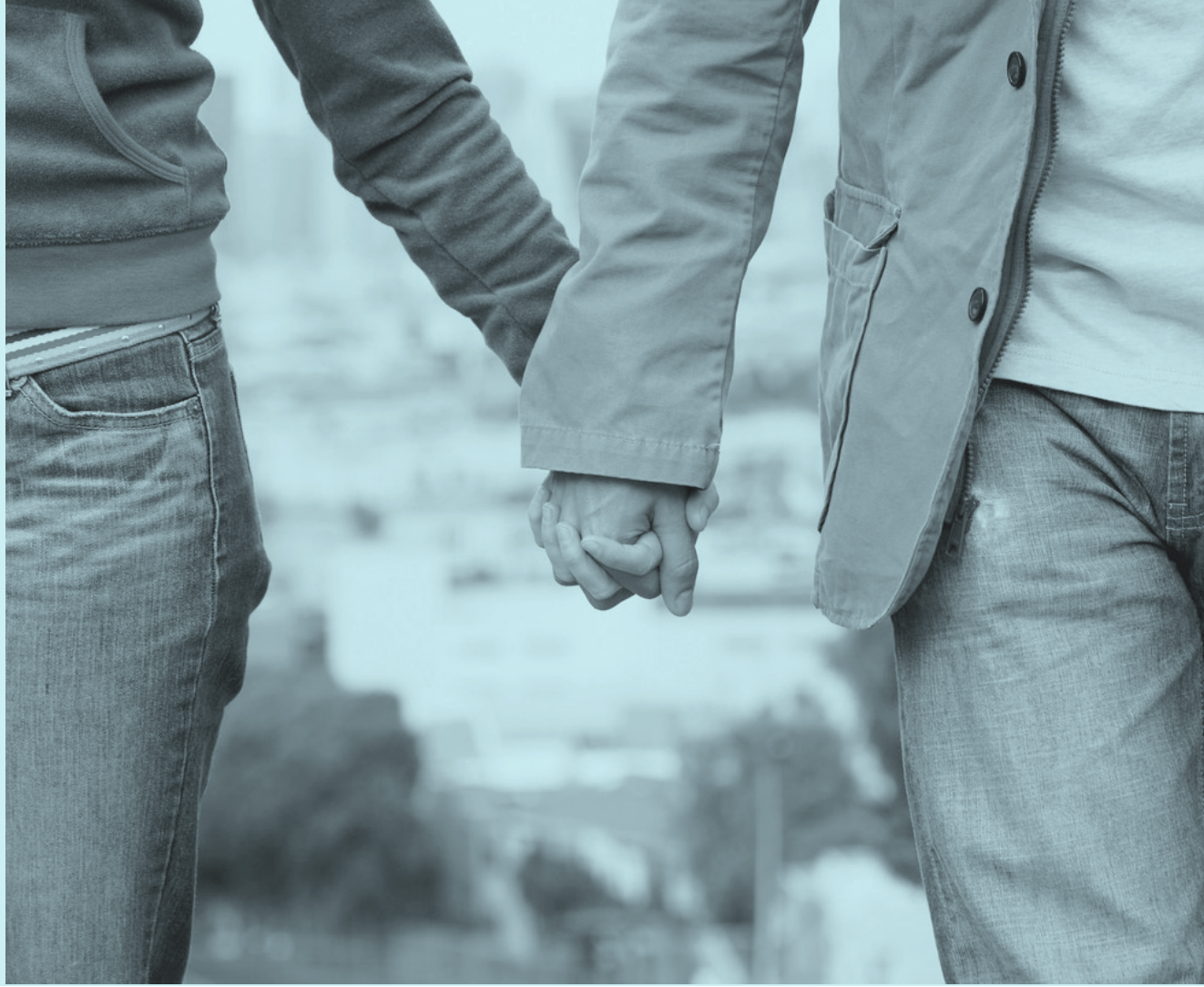

tas controladoras. La violencia genera efectos que pueden reproducir conductas en sentido negativo y extenderse a todos los contextos donde interactúa el adolescente, del ámbito privado trasciende al público. ${ }^{(5)}$

\section{Ciclos de la violencia en el noviazgo}

El patrón de abuso que sufren mujeres jóvenes en una relación de pareja es similar al que sufre una mujer maltratada adulta.

\section{Fase de acumulación de tensión. Se} manifiestan insultos, reproches, escenas de celos y control, silencios prolongados, burlas, malestar constante y en aumento.

2. Fase de estallido o explosión. Se manifiestan incidentes con todo tipo de agresión física, golpes, ruptura de objetos y amenazas.

\section{Fase de arrepentimiento o luna de} miel. Se manifiestan expresiones de perdón, promesas de cambio, interés sexual, regalos y "tranquilidad". El abuso severo se alterna con devoción y amor en esta fase, que resulta de gran atracción para los jóvenes debido a la actitud asumida por el agre- sor(a). Se transforma en un amante arrepentido(a), amable, que promete que no volverá a abusar. ${ }^{(5)}$

Cuando nos encontramos ante una relación donde la violencia persiste, este ciclo se repite, una y otra vez, donde la relación va construyendo una espiral y el período de duración entre las diferentes etapas es más rápido, modificándose el grado de violencia en cada fase. Por otro lado, la víctima se siente necesitada y valorada, asume la responsabilidad por la violencia y cree verdaderamente que el agresor(a) cambiará, sintiéndose culpable por haber pensado en dejarlo.(5)

La violencia en las relaciones de noviazgo, se presenta cuando ocurren actos que lastiman a la otra persona en el contexto de una relación en la que existe atracción y en la que los dos miembros de la pareja "salen". Se considera violencia psicológica a las agresiones verbales y emocionales, como intimidaciones, denigraciones, humillaciones y amenazas, entre otros. La violencia sexual, es el tercer tipo e incluye actos que atentan contra sus derechos sexuales y reproductivos, como las relaciones sexuales forzadas, la exposición a actividades 
sexuales indeseadas, el uso del sexo como forma de presión y manipulación y las críticas por el desempeño o la apariencia sexual. ${ }^{(6)}$

En la actualidad, los estudios relacionados sobre la violencia ya no solo se centran en matrimonios o la violencia del hombre hacia la mujer, sino se incluye la violencia en cualquier relación de pareja, incluyendo el noviazgo y en los jóvenes. Coinciden en que un alto porcentaje manifestó ser agredida por su pareja en el último año, además, no solo es la mujer la que sufre violencia sino que también hay contextos en los que la ejerce. (7-9) Otros estudios reportan que el perfil agresor es mayor en hombres (20.4\%), que en mujeres (13.4\%), diferencia que obliga a reflexionar sobre el hecho de que los estudiantes independientemente de su género violentan más de lo que son violentados ${ }^{(10)}$. La agresión psicológica predecía la agresión física y que esta última tendía a ser estable durante la historia de la relación. En México el $15.5 \%$ de las y los mexicanos entre 15 y 24 años con relaciones de pareja ha sido víctima de violencia física, un $75.8 \%$ ha sufrido agresiones psicológicas y un $16.5 \%$ ha vivido al menos una experiencia de ataque sexual. ${ }^{(1)}$

La violencia es un fenómeno complejo, ya que un tipo de violencia siempre genera otro tipo de violencia, por tal motivo requiere fuerza del estado entendido como la suma de instituciones de gobierno así como la ciudadanía. Desde el punto de vista de la salud mental, el personal de enfermería, debe abordar este problema no solo para conocer su prevalencia, si no para poder establecer estrategias encaminadas a prevenir las todas las formas de violencia que se pueden generar en las relaciones personales ${ }^{(6)}$. El objetivo del presente estudio es identificar la prevalencia de violencia en las relaciones de noviazgo que presentan los estudiantes de una preparatoria de Hermosillo, Sonora.

\section{METODOLOGÍA}

El diseño de la investigación es de tipo exploratoria, descriptiva y transversal. La población considerada en este estudio son los alumnos de la preparatoria pública de Sonora de primero, tercero y quinto semestre del turno matutino, la muestra fue seleccionada a través del proceso aleatorio simple, con un total de 214 alumnos encuestados.

Entre los criterios de inclusión se consideraron todos los estudiantes del plantel que aceptaron participar, alumnos de primero, tercero y quinto semestre del plantel y que tengan o hayan tenido una relación. Se excluyeron a aquellos estudiantes que no aceptaron participar en el estudio, que no hayan tenido alguna relación de pareja y a los que estaban casados.

El instrumento que se aplicó son del manual del taller Noviazgo entre adolescentes ${ }^{(11)}$, validado por Instituto Aguascalientes de las Mujeres, tiene como objetivo central identificar si los estudiantes del plantel sufren de violencia en el noviazgo, que tipo de violencia es la que sufren y como perciben los alumnos que afecta esta situación a su desempeño académico. Además, se apegó a lo dispuesto en el reglamento de la Ley General de Salud en Materia de Investigación para la Salud ${ }^{(12)}$

El análisis estadístico se llevó a cabo mediante el software (SPSS, versión 20) a través de estadística descriptiva como son medidas de tendencia central (media, mediana y desviación estándar) y a través tablas de frecuencia y porcentajes, gráficos de pastel y de barra.

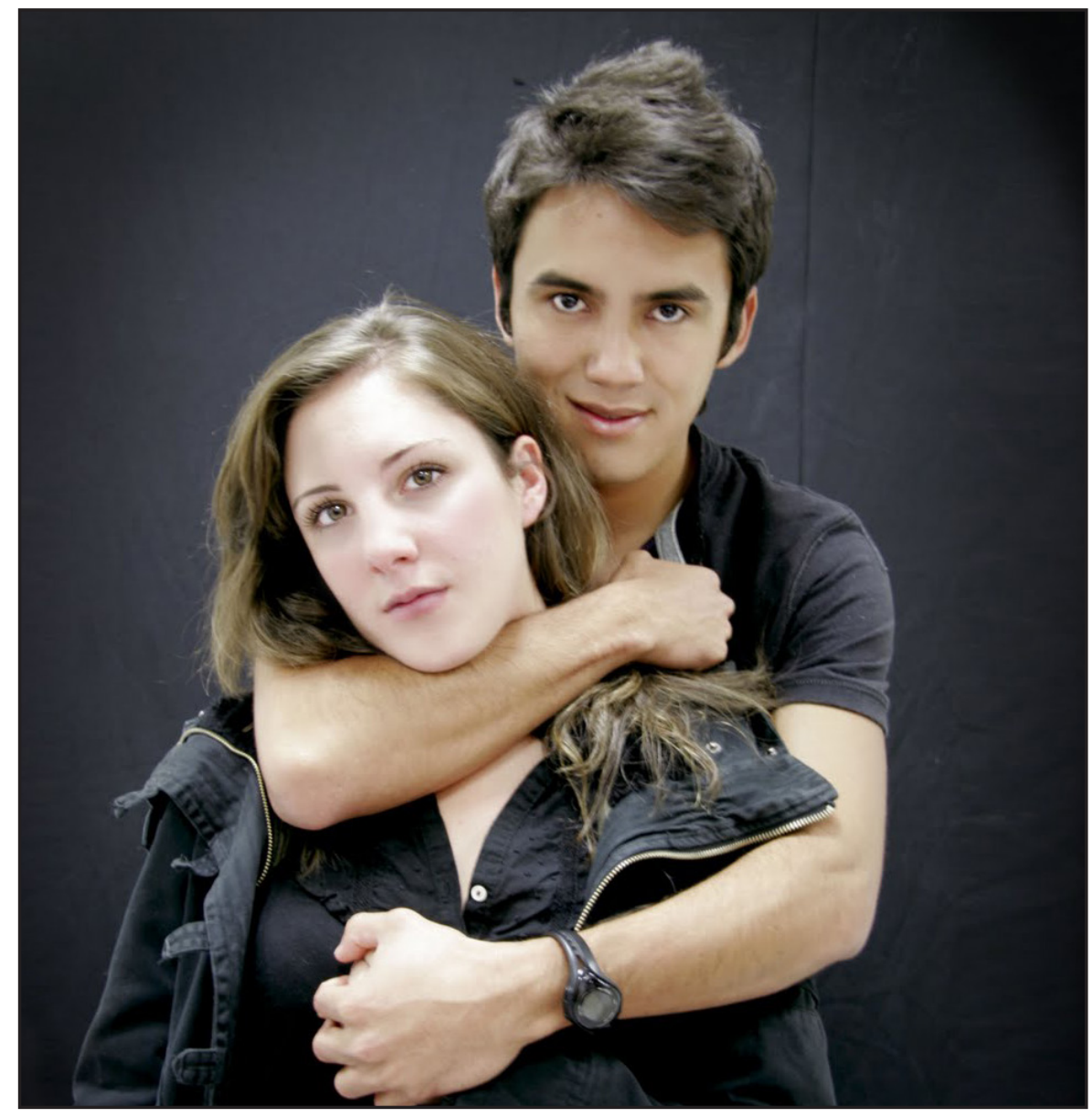




\section{RESULTADOS}

La mayoría de la muestra fue de género masculino con un porcentaje de $51.4 \%$ y el género femenino con un $48.6 \%$, como se muestra en la gráfica 1 .

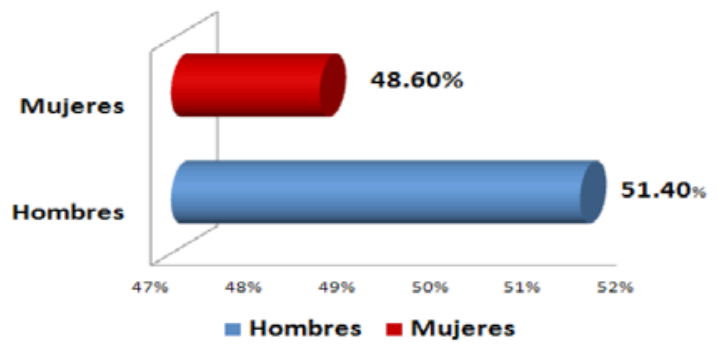

Gráfica 1. GÉNERo de los estudiantes del CONALEP, Hermosillo II. FUENTE: ENCUESTA DIRECTA N=214

Del $100 \%$ de la muestra el $52.3 \%$ se encuentra en una relación amorosa y el $47.6 \%$ se encuentran solteros (Gráfica 2).

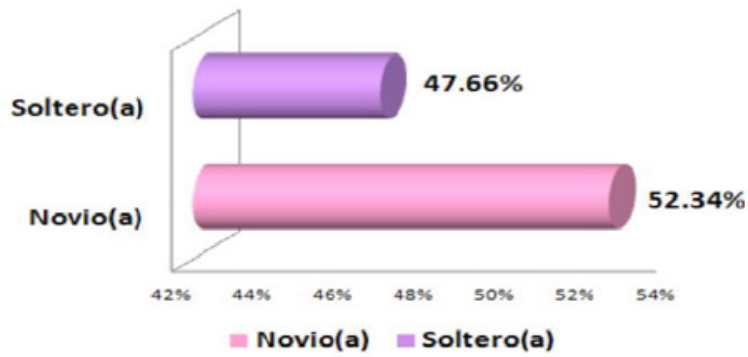

Gráfica 2. SituaCión SENTIMENTAL ACTUAL, ALUMNOS DEL PLANTEL CONALEP, HeRmosillo II. Fuente: ENCUESTA DIRECTA N=214

El $44.3 \%$ presentan algún grado de violencia. De estos jóvenes el $29.4 \%$ indican señales iniciales de violencia, otro $10.7 \%$ se identificaron con una relación de abuso en el noviazgo, mientras que el $3.3 \%$ mencionó estar en una relación de abuso severo, el $0.9 \%$ restante presentan relación de noviazgo violenta. Tabla 1.

\begin{tabular}{lc}
\hline Grado de violencia & $\%$ \\
\hline Sin violencia & 55.7 \\
\hline Señales iniciales de violencia & 29.4 \\
\hline Relación de abuso en le noviazgo & 10.7 \\
\hline Abuso severo & 3.3 \\
\hline Relación de noviazgo violenta & 0.9
\end{tabular}

Tabla 1. Grado de violencia en el noviazgo, alumnos del Plantel CONALEP, HERMOSILlo II. FUENTE: ENCUESTA DIRECTA N=214

En la gráfica 3, se muestra que el $55.6 \%$ se encuentra en una relación que no presenta violencia. Sin embargo, el $29.4 \%$ presentó señales iniciales de violencia, un $10.7 \%$ se encuentra en una relación de abuso, el $3.2 \%$ de los estudiantes encuestados están en una relación de abuso severo y el $0.9 \%$ presenta una relación violenta.

En cuanto a la violencia respecto a género, muestra un predominó en la de tipo emocional dirigida hacia las mujeres, pero también en una proporción importante a los hombres; seguida por la sexual que afecta mayormente a los hombres, y por último se encuentra el tipo de violencia física, como se mencionan en la tabla 2 y gráfica 4 .

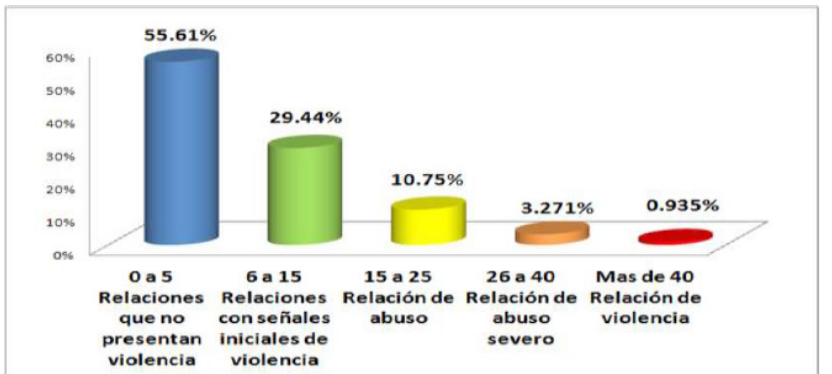

Gráfica 3. Grado de violencia, alumnos del plantel CONALEP, HerMOSILLO II. FUENTE: ENCUESTA DIRECTA N=214

\begin{tabular}{|c|c|c|c|}
\hline Tipo de Violencia & Género & fr & $\%$ \\
\hline \multirow{2}{*}{ Emocional } & Hombres & 51 & 23.5 \\
\cline { 2 - 4 } & Mujeres & 67 & 31.6 \\
\hline \multirow{2}{*}{ Física } & Hombres & 4 & 1.8 \\
\hline \multirow{2}{*}{ Sexual } & Mujeres & 10 & 4.5 \\
\cline { 2 - 4 } & Hombres & 62 & 29 \\
\cline { 2 - 4 } & Mujeres & 9 & 4
\end{tabular}

Tabla 2. Violencia Respecto al géNero, alumnos del Plantel CONALEP, HeRMOSILLO II. FUENTE: ENCUESTA DIRECTA N=214

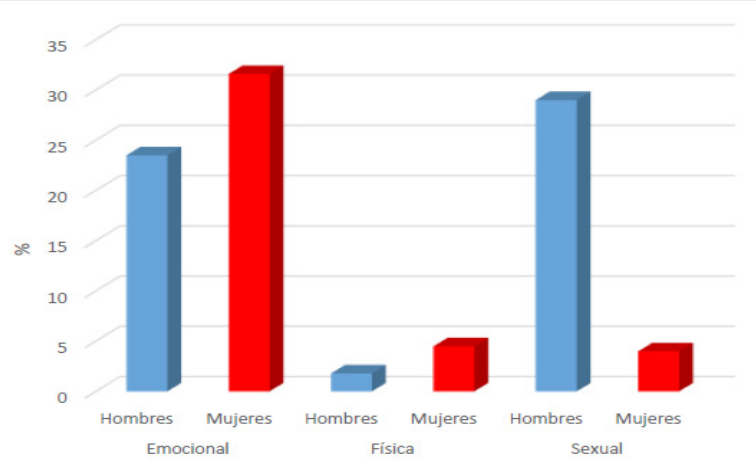

Gráfica 4. Violencia respecto al género, alumnos del plantel CONALEP, HERMOSILLO II. FUENTE: ENCUESTA DIRECTA N=214

\section{RECOMENDACIONES:}

La enfermería tiene un papel fundamental para intervenir en la violencia en el noviazgo, por su posición estratégica y la percepción que tienen las víctimas acerca del trabajo enfermero, por lo tanto sugerimos la implementación de consultorios de enfermería en las escuelas para la atención de problemas estudiantiles donde se vean aspectos tanto de salud física como mental.

De acuerdo a la detección de violencia en el noviazgo se puede implementar consejería de cómo seleccionar a la pareja correcta, que tipos de tratos se deben de tener para posterior mente evitar familias disfuncionales.

Debido a que el proceso enfermero es individualizado, cada caso será diferente al resto, por lo que no existe una intervención estandarizada para las personas que tengan este tipo de problema, ya que no todas ellas la padecen de la misma manera. Es por eso que los profesionales de enfermería deben conocer todos aspectos de este proceso y hacer un abordaje correcto del mismo según sea el caso que se esté presentando. 
También recomendamos realizar talleres dirigidos al cuidado de enfermería que actúen como prevención primaria y secundaria en los adolescentes y jóvenes para la violencia de tipo psicológica, física y sexual en las relaciones de noviazgo en los diferentes niveles de educación principalmente en secundaria y preparatoria, donde se resuelvan las situaciones de conflicto entre las parejas de forma no violenta a través del diálogo y negociación donde tomen acuerdos ambas partes de la pareja.

Se sugieren las siguientes intervenciones de enfermería:

4420 Acuerdo con el paciente
4470 Ayuda en la modificación
de sí mismo
4480 Facilitar la autoresponsabilidad
4700 Reestructuración cognitiva
4920 Escucha activa
5210 Guía de anticipación
5240 Asesoramiento
5250 Apoyo en la toma de decisiones
5270 Apoyo emocional
5340 Presencia
5390 Potenciación de la conciencia de sí
mismo
5430 Grupo de apoyo
5450 Terapia de grupo
5480 Clarificación de valores

4420 Acuerdo con el paciente 4470 Ayuda en la modificación de sí mismo

4480 Facilitar la autoresponsabilidad

4700 Reestructuración cognitiva

4920 Escucha activa

5210 Guía de anticipación

5240 Asesoramiento

5250 Apoyo en la toma de decisiones

5270 Apoyo emocional

5340 Presencia

5390 Potenciación de la conciencia de sí mismo

5430 Grupo de apoyo

5480 Clarificación de valores
CONCLUSIÓN

El noviazgo representa los sentimientos de "amor" que hay entre dos personas, pero además de compartir este tipo de sentimiento, así como existen sentimientos positivos hay negativos. Cuando una de las dos personas recibe o da maltrato durante la relación, existe violencia en el noviazgo. Muchos de los jóvenes carecen de información acerca de los síntomas de esta problemática es ahí donde confunden sus propias conductas, y alteran el trato hacia su pareja.

Algunos de los factores que desencadenan la violencia en el noviazgo esta: antecedente de violencia en el hogar o con las personas más cercanas o sencillamente con sus anteriores parejas, también el abuso de sustancias nocivas para la salud. Estos factores desatan conductas violentas hacia la otra persona y la pue- den dañar física o emocionalmente.

No siempre el enojo o la violencia es la mejor respuesta a los problemas en el noviazgo, el aprender a controlarse y buscar mejor opciones de los problemas, es la mejor solución. La comunicación es uno de los factores más importantes que no debe ser reprimida por la pareja, al intercambiar diferentes gustos, ideas, intereses, y valores contribuyes a construir una relación sana sobre todo respetuosa con esa persona.

En la presente investigación realizada en la preparatoria publica de Sonora, la mayoría de los hombres fueron los que sufren de algún tipo de violencia, es por esta razón hay que actuar, educando a la población, con mayor énfasis a los adolescentes, por la etapa de desarrollo y de aprendizaje donde se encuentran.

\section{REFERENCIAS BIBLIOGRAFICAS}

1. Violencia contra la mujer. [Internet]. 2013. Available from: http://www.who.int/mediacentre/factsheets/fs239/es/.

2. Pública SdS. Guía del Taller Prevención de la Violencia en el Noviazgo. 2012. Available from: http://www.ssp.gob.mx/ portalWebApp/ShowBinary?nodeId =/ BEA\%20Repository/1214154//archivo.

3. Encuesta Nacional sobre la Dinámica de las Relaciones en los Hogares 2011 [Internet]. 2011. Available from: http://www. inegi.org.mx/est/contenidos/Proyectos/ Encuestas/Hogares/especiales/endireh/ endireh2011/default.aspx.

4. Furman W, Brown BB, Feiring C. The development of romantic relationships in adolescence: Cambridge University Press; 1999.
5. de Weiss-Vargas-Trujillo $P$, de Weiss $S P$, Vargas-Trujillo E, Guerrero IP. Yo, Adolecente: Respuetas Claras A Mis Grandes Dudas: Planeta; 2000.

6. Rey-Anacona CA. Maltrato de tipo físico, psicológico, emocional, sexual y económico en el noviazgo: un estudio exploratorio. Acta colombiana de psicología. 2009;12(2):27-36.

7. Straus MA. Prevalence of violence against dating partners by male and female university students worldwide. Violence against women. 2004;10(7):790-811.

8. Sears HA, Byers ES, Price EL. The co-occurrence of adolescent boys' and girls' use of psychologically, physically, and sexually abusive behaviours in their dating relationships. Journal of adolescence. 2007;30(3):487-504.
9. Control $C f D$, Prevention. Physical dating violence among high school students--United States, 2003. MMWR: Morbidity and mortality weekly report. 2006;55(19):532-5.

10. O'Leary KD, Smith Slep AM. A dyadic longitudinal model of adolescent dating aggression. Journal of Clinical Child and Adolescent Psychology. 2003;32(3):31427.

11. Guzmán EC. Manual del taller: Noviazgo entre adolecentes 2000. Available from: http://cedoc.inmujeres.gob.mx/insp/taller_noviazgo.pdf.

12. Salud Sd. REGLAMENTO de la Ley General de Salud en Materia de Investigación para la Salud In: Salud Sd, editor. 1987 